\title{
Characterization of diamagnetism inside an ECR thruster with a diamagnetic loop
}

Cite as: Phys. Plasmas 26, 053511 (2019); https://doi.org/10.1063/1.5093980

Submitted: 26 February 2019. Accepted: 07 May 2019. Published Online: 29 May 2019

S. Correyero (D), M. Merino (D), Paul-Quentin Elias (D), J. Jarrige, D. Packan, and E. Ahedo (D)

\section{ARTICLES YOU MAY BE INTERESTED IN}

E×B configurations for high-throughput plasma mass separation: An outlook on possibilities and challenges

Physics of Plasmas 26, 043511 (2019); https://doi.org/10.1063/1.5083229

Boundary-induced effect on the spoke-like activity in E×B plasma

Physics of Plasmas 26, 053503 (2019); https://doi.org/10.1063/1.5092702

Radio frequency wave interactions with a plasma sheath: The role of wave and plasma sheath impedances

Physics of Plasmas 26, 052503 (2019); https://doi.org/10.1063/1.5088343

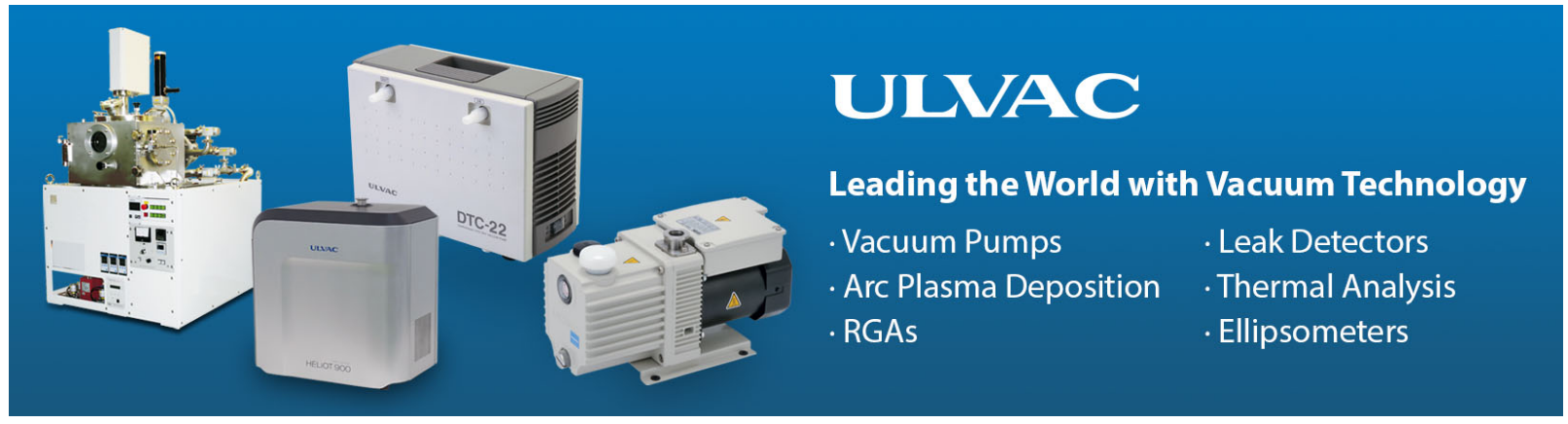




\title{
Characterization of diamagnetism inside an ECR thruster with a diamagnetic loop
}

\author{
Cite as: Phys. Plasmas 26, 053511 (2019); doi: 10.1063/1.5093980 \\ Submitted: 26 February 2019 - Accepted: 7 May 2019. \\ Published Online: 29 May 2019
}

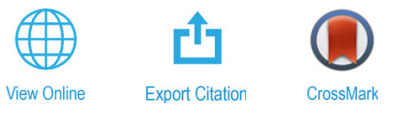

\author{
S. Correyero, ${ }^{1, a)}$ (D) M. Merino, (D) Paul-Quentin Elias, ${ }^{2}$ (D) J. Jarrige, ${ }^{2}$ D. Packan, ${ }^{2}$ and E. Ahedo ${ }^{7}$
}

\section{AFFILIATIONS}

${ }^{7}$ Equipo de Propulsión Espacial y Plasmas, Universidad Carlos III de Madrid, Leganés 28911, Spain

${ }^{2}$ ONERA, The French Aerospace Laboratory, Palaiseau, Paris 91120, France

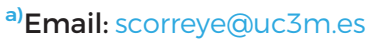

\begin{abstract}
The plasma-induced magnetic field in an electron cyclotron resonance plasma thruster is measured non-intrusively by means of a diamagnetic loop that encloses the plasma flow. The calibration process is described, and parasitic currents in the thruster walls and plasma oscillations are identified as the dominant sources of uncertainty. The integrated magnetic flux is seen to depend on the applied power and less significantly on the mass flow rate. The effect of the diamagnetic loop radius is also studied by testing two loops of different diameters. To estimate the perpendicular electron pressure in the plasma from the loop measurements, two plasma beam models, 1D and 2D, are used. While both models give similar results for the small loop, they differ significantly for the large loop, showing the relevance of $2 \mathrm{D}$ effects when a large diamagnetic loop is used.
\end{abstract}

Published under license by AIP Publishing. https://doi.org/10.1063/1.5093980

\section{INTRODUCTION}

Electrodeless plasma thrusters, such as an electron cyclotron resonance thruster (ECRT) ${ }^{1-5}$ and a helicon plasma thruster (HPT), ${ }^{6-8}$ produce a cold-ion, hot-electron plasma by applying electromagnetic radiation to ionize and heat the propellant. An externally applied magnetic field $\boldsymbol{B}_{a}$ confines the plasma away from the lateral walls of the thruster chamber and enables the propagation of the electromagnetic radiation into the plasma, where it is absorbed. Outside of the thruster, $\boldsymbol{B}_{a}$ diverges to form a magnetic nozzle (MN), where the plasma is expanded and accelerated quasineutrally by Lorentz forces. ${ }^{9,10}$ In the typical operation regime, electrons are well magnetized in the $\mathrm{MN}$ and follow the magnetic lines, whereas ions are, in general, weakly magnetized. As the electrons expand downstream, an ambipolar electric field $\boldsymbol{E}$ arises in the plasma that confines most electrons and accelerates ions, converting the electron thermal energy into directed kinetic ion energy and thus creating a supersonic plasma jet channeled by the nozzle. Only the most energetic electrons overcome the potential barrier to create a globally current-free plasma plume. Finally, the plasma detaches from the closed lines of the applied field to form a free plasma plume. ${ }^{11}$

Thrust can be decomposed into pressure thrust and magnetic thrust. The former is due to the plasma pressure on the chamber walls and is considered an inefficient thrusting mechanism due to the recombination losses it entails. The latter is the reaction to the magnetic acceleration of the expanding plasma, which takes place mainly in the MN region. Positive magnetic thrust requires the plasma to be diamagnetic so that the magnetic force between the plasma and the magnets or coils of the thruster is repulsive: microscopically, the cyclotron motion of each individual magnetized electron creates a diamagnetic field that opposes the applied one and experiences a force that pushes it in the downstream direction due to the magnetic mirror effect. Macroscopically, the sum of all electron gyrations and drifts gives rise to an azimuthal diamagnetic electron current density $j_{\theta e}$ that interacts with $\boldsymbol{B}_{a}$ to create an inward-confining Lorentz force that balances the outward-expanding perpendicular electron pressure $p_{\perp e}$. In the divergent $\mathrm{MN}$, part of this Lorentz force density $\left(j_{\theta e} B_{r}\right)$ acts in the axial direction. The reaction to this force is the largest contribution to magnetic thrust since the ion azimuthal current $j_{\theta i}$ (which can incidentally be paramagnetic and hence generate magnetic drag) is small in the cases of interest. ${ }^{9}$ The azimuthal electron current $j_{\theta e}$ is also the main contributor to the plasma-induced magnetic field $\boldsymbol{B}_{p}$, which opposes $\boldsymbol{B}_{a}$, tends to demagnetize the plasma in the $\mathrm{MN}$, and increases the divergence of the $\mathrm{MN} .^{12}$

Magnetic thrust scales with the perpendicular electron pressure $p_{\perp e}$ at the source, and it is thus desirable to maximize its value during plasma production. In particular, ECRTs use microwaves that resonate with the electron cyclotron frequency in certain regions of the device, depositing most of the electromagnetic power into the electron perpendicular motion at resonance and resulting in a partially anisotropic 
electron distribution function, depending on the collisionality of the plasma. ${ }^{13}$ Hence, a larger magnetic-to-pressure thrust ratio is expected in these thrusters. Indeed, while isotropic electron populations give rise to about $50 \%$ of the total thrust being generated at the $\mathrm{MN},{ }^{9}$ preliminary measurements with an ECRT prototype show that this fraction can be as large as $80 \% .{ }^{14}$ This makes the MN of the ECRT an even more essential component for the operation of the thruster.

As $\boldsymbol{B}_{p}$ is generated by $j_{\theta e}$, measuring $\boldsymbol{B}_{p}$ allows estimating $p_{\perp e}$, which can provide important information about the operation and performance of these thrusters and eventually on the magnetic contribution to the thrust. A well-established diagnostic technique to measure $\boldsymbol{B}_{p}$ is the diamagnetic loop, which consists of a coil of several turns wound around the plasma jet. Its working principle is simple and relies on the measurement of the induced voltage caused by a change in the magnetic flux enclosed by the loop: ${ }^{15}$ obeying Faraday's law, the voltage at the loop ends can be time-integrated during the thruster shutdown transient to obtain the total plasma-induced magnetic flux $\boldsymbol{B}_{p}$ across the loop that exists just before plasma extinction. The integrated voltage signal is proportional to the number of turns of the loop, which must be chosen according to the sensitivity of the acquisition system.

In the 1960s, the diamagnetic loop was used in the magneticconfinement fusion community to quantify the perpendicular plasma pressure in a reactor. ${ }^{16-18}$ In the field of electric propulsion, Sercel first attempted to use a diamagnetic loop in the 1990s to estimate the plasma pressure inside an ECRT, but his efforts at that time led to inconclusive results, ${ }^{19}$ allegedly due to significant systematic errors in the measurements. More recently, Ando et al. measured the change in $\boldsymbol{B}_{p}$ and the plasma thermal energy in a magneto-plasma-dynamic thruster when switching on an additional ion cyclotron heating stage. ${ }^{20}$ Regarding Hall Effect Thrusters, diamagnetic loops have been used to measure the azimuthal drift current in the channel discharge by fast current interruption techniques. ${ }^{21,22}$ Besides these noteworthy studies and to the best knowledge of the authors, large diamagnetic loops that embrace the plasma flow have barely been applied in electric propulsion research. More commonly, the plasma-induced magnetic field $\boldsymbol{B}_{p}$ in plasma thrusters has been measured locally using intrusive Hall probes and B-dot probes into the plasma plume. ${ }^{23,24}$

In other fields of plasma physics, diamagnetic loops have been used to investigate the plasma-induced magnetic field in various plasma devices. Stenzel and Urrutia studied MHD turbulence in a high- $\beta$, dense plasma discharge and the expulsion of the magnetic field using a diamagnetic loop and a $3 \mathrm{D}$ magnetic probe. ${ }^{25}$ Corr and Boswell investigated diamagnetism in a helicon source discharge by placing diamagnetic loops around the channel and the plasma core. ${ }^{26}$ As a last example, Noland et al. employ a diamagnetic loop on an electron-cyclotron resonance ion source to measure $\boldsymbol{B}_{p}$ and then use a 1D MHD model to estimate the mean plasma pressure. ${ }^{2}$

The present work presents a parametric investigation of the operation of an ECRT prototype developed at ONERA, France, using diamagnetic loop measurements with two loops of different diameters placed around the plasma jet. The integrated induced magnetic flux is then used to evaluate the perpendicular electron pressure inside the plasma jet, and finally, the influence of $2 \mathrm{D}$ effects, especially on the measurements of the loop with a larger diameter, is pointed out.

The rest of this article is structured as follows. The experimental setup is explained in Sec. II. The calibration of the diamagnetic loops is detailed in Sec. III. Section IV presents the experimental results of the induced magnetic flux at various thruster power levels and mass flow rates. Section $V$ estimates the perpendicular electron pressure from the induced magnetic flux measurements with two models: a simple analytical 1D model which considers an infinite uniform magnetized plasma column in equilibrium and a 2D MN model developed by Ahedo and Merino. ${ }^{9}$ The $2 \mathrm{D}$ effects on the measurements are also discussed there. The main conclusions of this work are gathered in Sec. VI.

\section{EXPERIMENTAL SETUP \\ A. ECRT prototype}

The thruster prototype consists of a coaxial structure composed of an inner stainless-steel rod $(0.9 \mathrm{~mm}$ radius $)$ surrounded by a $15 \mathrm{~mm}$ long and $13.5 \mathrm{~mm}$ radius cylinder into which $2.45 \mathrm{GHz}$ electromagnetic waves propagate. The back plate of the thruster is a cylindrical piece of $3 \mathrm{~mm}$ length made of boron nitride while the lateral thruster walls are made of graphite. A Neodymium permanent magnet creates a static magnetic field in such a way that electrons reach the cyclotron resonance condition (at 875 Gauss for $2.45 \mathrm{GHz}$ ) inside the source. The magnetic field generated by the magnet is also responsible for the fully-divergent magnetic nozzle topology (see Fig. 1), simplifying its design and construction. Finally, the propellant (xenon in the present case) is injected through two symmetrical holes of $1 \mathrm{~mm}$ diameter at the back plate.

\section{B. Electronics and vacuum facility}

Microwave power at $2.45 \mathrm{GHz}$ is transmitted to the thruster from a solid-state amplifier from Kuhne Electronics (100 W maximum power) through a $50 \Omega$ coaxial line, whose losses have been characterized using a vector network analyzer. The output of the generator is connected to a circulator, which allows us to dissipate the reflected power in a $50 \Omega$ load. Before entering the vacuum chamber, a bidirectional coupler measures the forward and reflected power through two calibrated diodes, whose values are registered through a $1 \mathrm{GHz}$ Oscilloscope. The uncertainty of the power measurement is $\pm 1 \mathrm{~W}$.

The tests carried out in this work have been performed at ONERA facilities, located in Palaiseau (France). The vacuum chamber consists of a cylindrical vessel of $2 \mathrm{~m}$ length and $0.8 \mathrm{~m}$ diameter. Three turbomolecular pumps and one cryogenic pump constitute the pumping system which has a total pumping speed of $13000 \mathrm{l} / \mathrm{s}$ of Xenon and can reach a background pressure of $10^{-7} \mathrm{mbar}$. The background chamber pressure while operating at $2 \mathrm{sccm}$ of xenon was $3.7 \times 10^{-6} \mathrm{mbar}$.

A direct-current blocker is installed between the power line and the thruster to ensure it is electrically isolated. The thruster floating potential while operating is monitored by means of a multimeter. Figure 2 shows a schematic of the vacuum setup and the power line.

\section{Diamagnetic loops}

To measure the diamagnetic signal inside the ECR thruster, two diamagnetic loops of different sizes were built:

1. Loop A: $D_{A}=3 \mathrm{~cm}$ diameter, $L_{A}=1.5 \mathrm{~cm}$ length, $N_{A}=30$ turns. 2. Loop B: $D_{B}=30 \mathrm{~cm}$ diameter, $L_{B}=3 \mathrm{~cm}$ length, $N_{B}=12$ turns.

Both loops are made of insulated copper wire. A $1 \mathrm{~mm}$ teflon layer separates Loop A from the thruster walls, while Loop B is positioned with a plastic holder. The diamagnetic loops are fixed in the setup and installed coaxially with the thruster walls. Loop A is wrapped exactly around the thruster walls while the half length of 
(a)

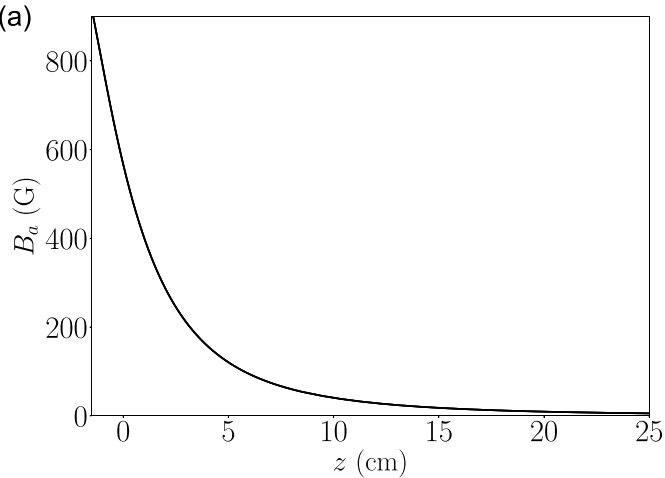

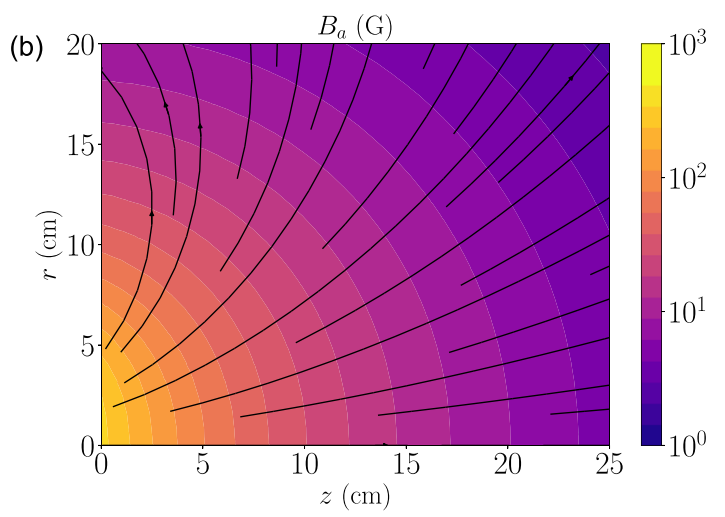

FIG. 1. (a) Magnitude of the applied magnetic field $\boldsymbol{B}_{a}$ at the thruster axis ( $z=0$ corresponds to the exit plane) and (b) 2D magnetic field topology.

Loop B coincides with the thruster exit plane. Figure 3 shows an schematic of the position of the loops.

The diamagnetic time signal of each loop is measured by a $1 \mathrm{GHz}$ oscilloscope during the transient shutdown of the plasma, starting from a steady-state operation point. Then, the time integration of the Faraday law for the magnetic induction yields:

$$
\int_{0}^{t} V d t=-N_{L} \cdot \Phi_{p},
$$

where $V$ is the potential difference between the loop terminals, $N_{L}$ is the number of turns of the loop, and $\Phi_{p}$ is the variation of magnetic flux enclosed by the loop before and after the shutdown. Since the applied magnetic field is generated by a permanent magnet, the magnetic flux measured by the loop corresponds only to the induced plasma magnetic field.

\section{DIAMAGNETIC SIGNAL CALIBRATION}

Identifying potential sources of error in the measurement of the voltage signal at the loop terminals is essential to guarantee a valid computation of the plasma-induced magnetic flux. Particularly important are the eddy currents through the thruster walls, since a cylindrical conductive material is enclosed by the diamagnetic loop. Other effects as the thruster potential drop during the shutdown or possible parasite currents from all the electronic systems involved have also been characterized. This section analyzes these effects in the experiment.

\section{A. Effect of eddy currents}

During thruster shutdown, and as the total magnetic flux varies, eddy currents are induced in the conductive parts of the device. In particular, moderate eddy currents can appear in the conductive walls of

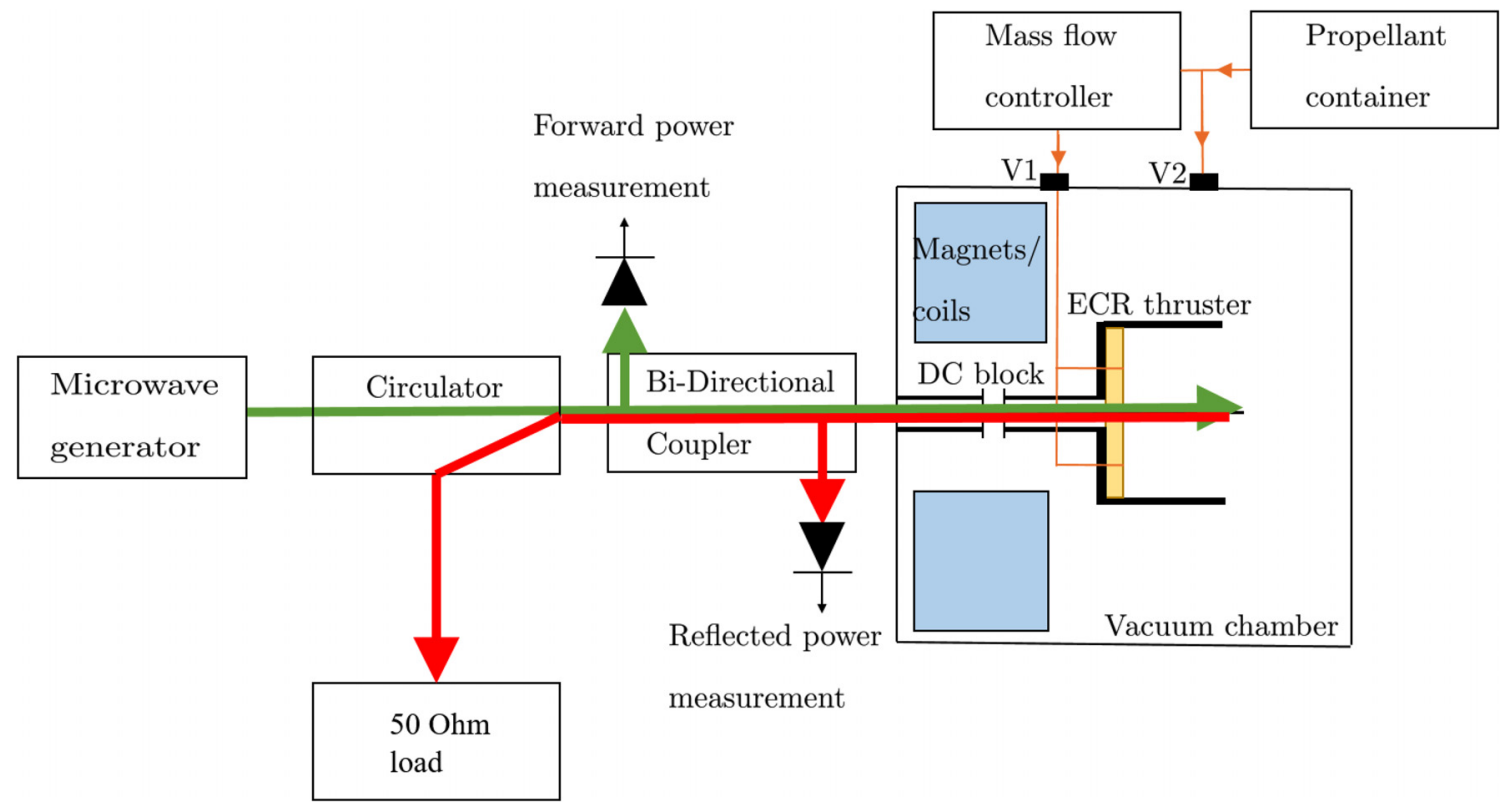

FIG. 2. Schematic of microwave power line and subsystems in the vacuum setup. 
$3 \mathrm{~cm}$

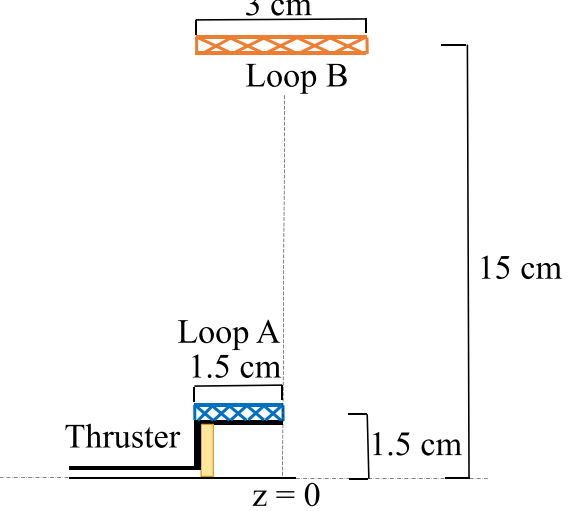

FIG. 3. Axisymmetric schematic of the thruster and the diamagnetic loops.

the thruster. These eddy currents create a magnetic field that opposes the field variation (Lenz's law), and therefore, they affect the flux variation measured by the loop. While the overall time integral of Eq. (1) is not modified once the eddy currents die away due to the resistivity of the material, this phenomenon weakens the signal to be integrated (it could even remain below the sensitivity of the acquisition system) and increases its decay time. Consequently, it could make the detection of the signal unaffordable.

To evaluate the influence of this effect, a characterization solenoid (length $L_{c}=200 \mathrm{~mm}$, number of turns $N_{c}=89$, and radius $R_{c}$ $=10 \mathrm{~mm}$ ) was built to emulate the voltage drop that is recorded by the diamagnetic loop when the thruster is powered off. This setup allows to study the eddy currents phenomena for a known input magnetic field and in ambient conditions. ${ }^{28}$ The solenoid was placed coaxially with the thruster walls and the diamagnetic loop (see Fig. 4).

By generating a pulsed current signal on the solenoid, the induced voltage in the diamagnetic loop was recorded. The current
Diamagnetic

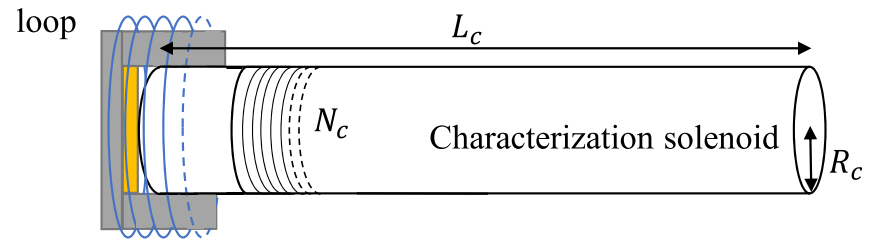

\section{ECR thruster}

FIG. 4. Schematic of the characterization solenoid placed coaxially with diamagnetic Loop $A$ and the thruster walls.

amplitude was controlled so that the response was comparable to the expected plasma diamagnetism. Figure 5 represents the electric circuit that was built to pulse the coil at specific values of current together with the diamagnetic loop circuit schematic. The loop and cable resistance $R_{D L}$ is negligible compared to the scope impedance. To illustrate the measurement characteristics of the system, the gain magnitude frequency response of loop A is plotted in Fig. 6 together with the input current impulse from the characterization solenoid. Notice that the system behaves as a low-pass filter with a power roll-off of $20 \mathrm{~dB} / \mathrm{dec}$ at high frequencies, with the cut-off at $150 \mathrm{kHz}$ (shown as a vertical dashed line in Fig. 6). For verification, the circuit has been simulated using the software LTspice to study the system response.

Two different materials were used to evaluate the effect of eddy currents in the thruster walls: graphite (the actual material of the present ECRT prototype walls) and aluminum. As can be observed in Fig. 7, the voltage integral could not be fully recovered in the case of aluminum walls due to the large time response of the eddy currents and the low value of the resulting diamagnetic signal. On the contrary, the lower conductivity of graphite resulted in a diamagnetic signal, comparable to the absence of conducting walls. The time integral of each curve corresponds to $7.53 \times 10^{-8} \mathrm{~V}$ s without thruster walls

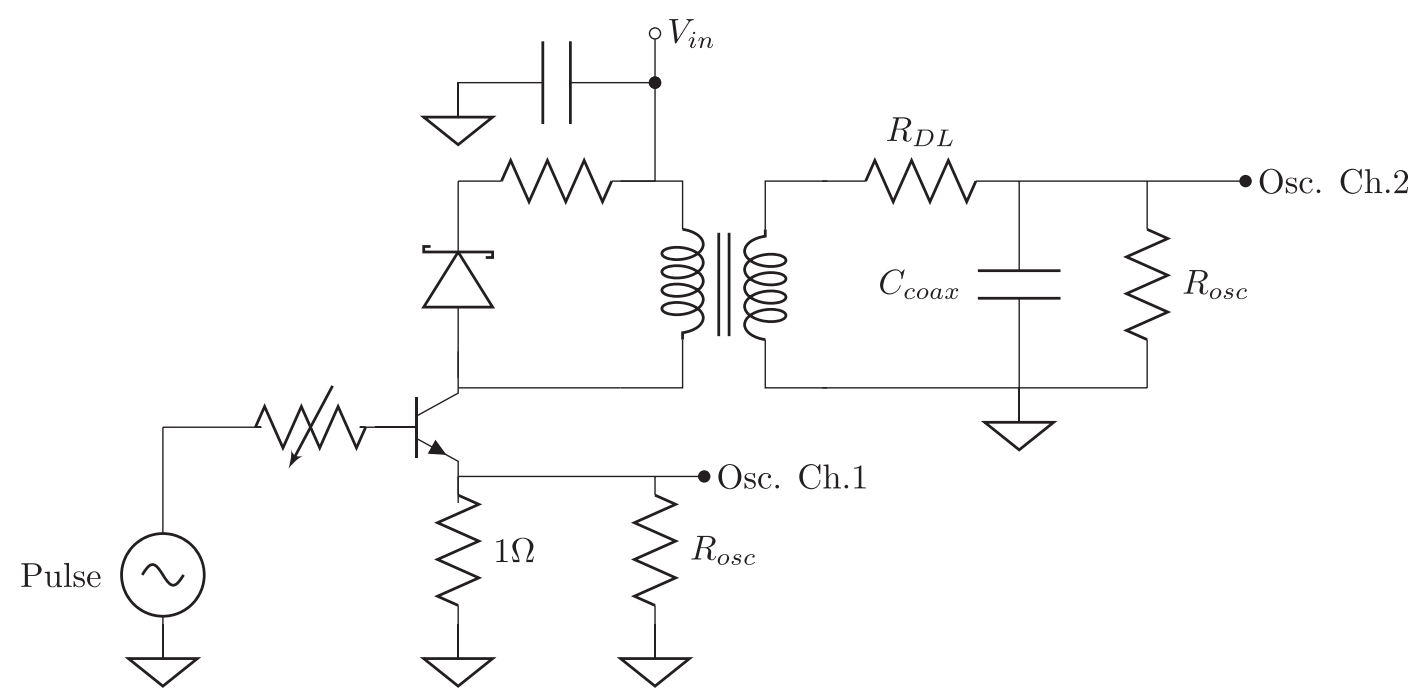

FIG. 5. Characterization solenoid circuit coupled with the diamagnetic loop circuit. $R_{\text {osc }}$ is the scope input impedance set on $50 \Omega, C_{\text {coax }}$ is the parallel capacitance mainly due to the coaxial cables, and $R_{D L}$ is the diamagnetic loop series resistance. The parallel capacitance can be estimated based on the capacitance of a RG-58 (BNC $50 \Omega$ ) cable, which in this case is around $300 \mathrm{pF}$. The diamagnetic loop circuit inductance has been estimated to be $50 \mu \mathrm{H}$ and $80 \mu \mathrm{H}$ for loops A and B, respectively. 

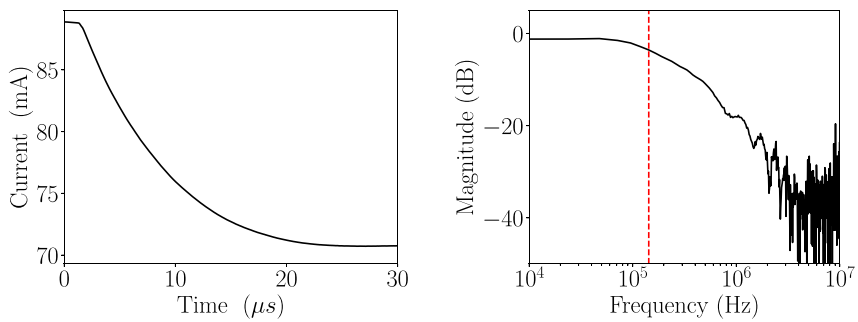

FIG. 6. Output signal from characterization solenoid (left) and the gain magnitude frequency response of the small diamagnetic loop (right), where the red dashed vertical line represents the cut-off frequency $(-3 \mathrm{~dB})$.

(and thus without eddy currents), $7.45 \times 10^{-8} \mathrm{~V} \mathrm{~s}$ for graphite walls, and $9.48 \times 10^{-9} \mathrm{~V} \mathrm{~s}$ for aluminum walls. With graphite walls, only a relative error of $1.1 \%$ on the total integral is committed, which is deemed acceptable for the present measurements. On the contrary, aluminum walls result in an error around $87 \%$. It should be noted that integrating over a larger time in this case does not improve significantly the result, since the background noise is above the signal itself after the initial instants.

Concerning eddy currents in the vacuum chamber walls, although the same phenomenon could in principle take place, no measurable effect was found. The calibration process was performed inside and outside the vacuum chamber to check the influence in the diamagnetic loop signal. In the case of "no thruster walls," the characterization solenoid was also used to compare the estimated magnetic flux and the real measured flux by the diamagnetic loop, finding a very good agreement between the theoretical and experimental values in the desired range, avoiding the need for a correction factor.

\section{B. Plasma oscillations}

To better understand the nature of the diamagnetic signal, a specific test was carried out to relate the plasma extinction process and the decay of the diamagnetic field. Together with the diamagnetic

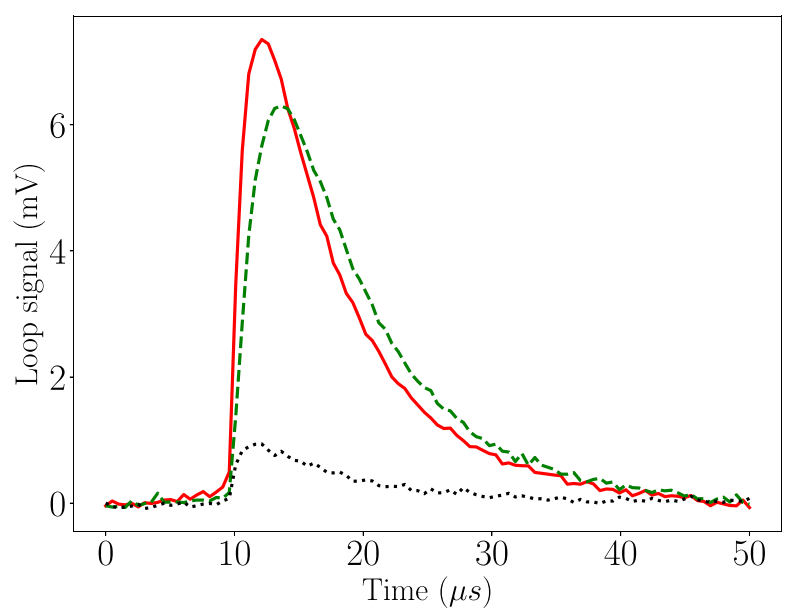

FIG. 7. Signal of the diamagnetic Loop A with the characterization solenoid. Three different cases are represented: aluminum thruster walls $(. . . .$.$) , graphite thruster$ walls (- - -), and no thruster (- $)$. signal, three different measurements were taken simultaneously: first, the microwave forward power entering the thruster line was read by means of a calibrated diode connected to a directional coupler; second, the thruster floating potential was directly measured by a voltage probe connected to the thruster walls; and finally, the light intensity coming from the plasma source was collected into an optic fiber and recorded using a photodiode.

Figure 8 (left) shows the normalized voltage at the diamagnetic loop and the three additional measurements, starting from operation at $30 \mathrm{~W}$ power and $3 \mathrm{sccm}$ Xenon. The simultaneity of the four signals illustrates the magnetic flux change at diamagnetic loop during the shutdown. Visibly, the three additional measurements are stable before shutdown. In contrast, the diamagnetic loop signal displays large oscillations, which could be possibly attributed to plasma instabilities during operation. These oscillations are an important source of error in the time integration of Eq. (1), generating uncertainty in the measurement of the magnetic flux change at shutdown. These oscillations have been characterized and used to define the error bars in Fig. 10. Their frequency and amplitude are operation point-dependent, but in most cases under study, peaks between $2 \mathrm{kHz}$ and $150 \mathrm{kHz}$ were identified. Incidentally, these oscillations are in the same frequency range as the ones found by Sercel in Ref. 19.

\section{Other effects}

Finally, the effect of the thruster potential decay on the diamagnetic loop signal during the shutdown was evaluated. Microwave power is transmitted to the plasma by a $50 \Omega$ coaxial line, and a Direct Current Blocker (DC Block) is placed between the line and the source cavity to isolate the generator and components from the plasma. Therefore, the conductive thruster walls can float and they reach high electric potentials (around 100-200 V) when the thruster is operating. This floating potential is maintained by the plasma and depends directly on the plasma properties (ion and electron fluxes, sheath between thruster and plasma, etc.) and the thruster materials. At the thruster shutdown, the floating potential drops to a residual value as

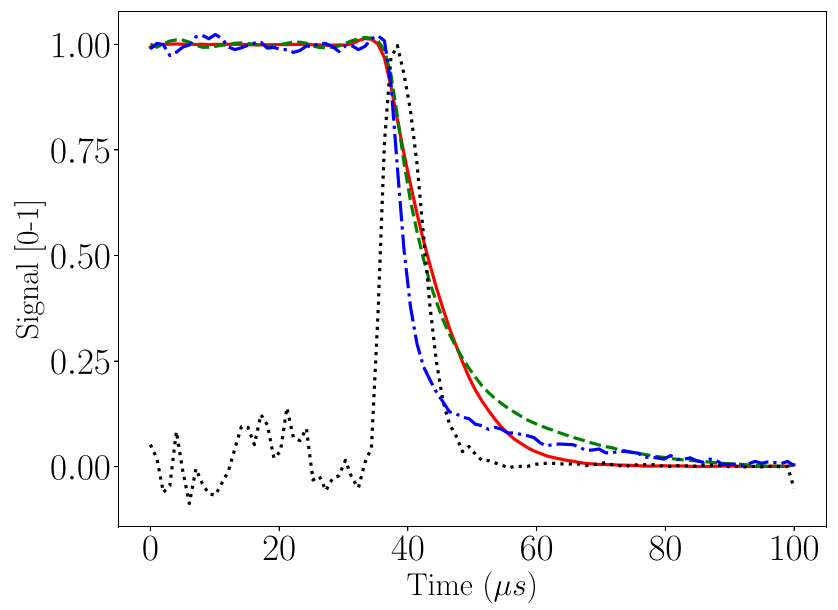

FIG. 8. Signal of diamagnetic Loop A (.....), thruster floating potential (- - -), forward power (-), and light intensity coming from the plasma source (--.--) during thruster shutdown, normalized. 

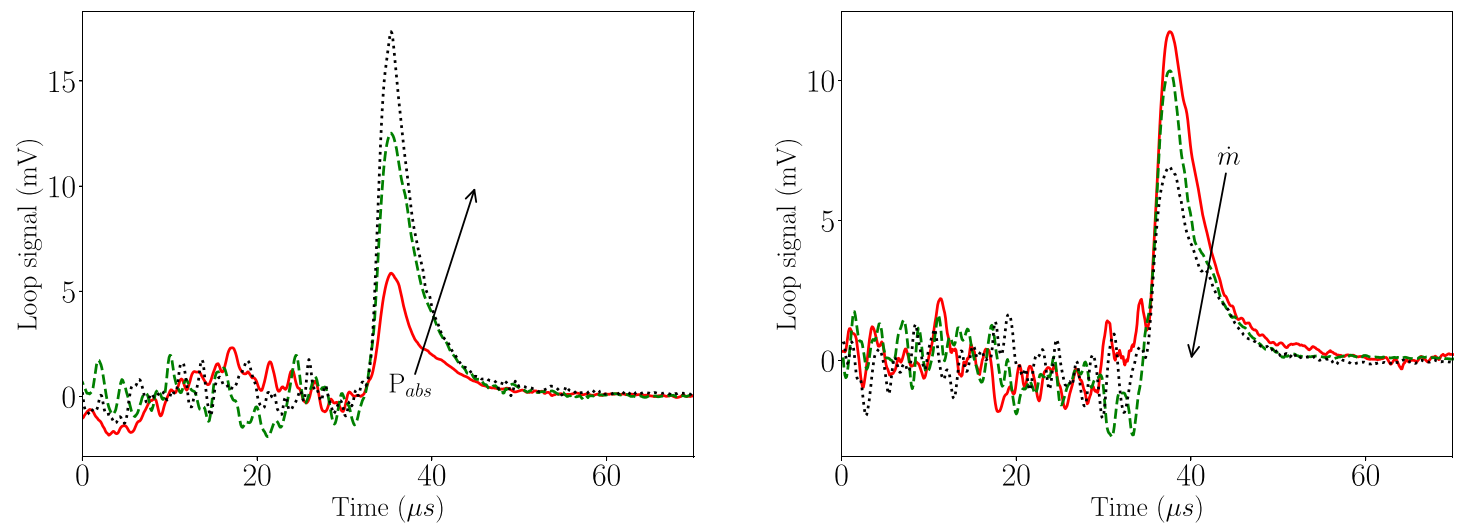

FIG. 9. Diamagnetic signal (in millivolt) vs time during the thruster shutdown for: (left) $2 \mathrm{sccm}$ of Xenon and different power levels: $15 \mathrm{~W}$ (- $-30 \mathrm{~W}(---)$, and $41 \mathrm{~W}$ (......), and (right) $30 \mathrm{~W}$ at three different xenon mass flow rates: 1 (-), $2(---)$, and $3 \mathrm{sccm}(\ldots . .$.$) .$

shown in Fig. 8. To study any possible influence of this electrostatic effect on the diamagnetic signal, the thruster was charged externally in the absence of plasma to $50 \mathrm{~V}$ and discharged rapidly simulating the thruster shutdown. The signal of the loop was recorded simultaneously with the thruster potential. The observed effect was negligible when compared with the total signal from the diamagnetic loop.

\section{PLASMA-INDUCED MAGNETIC FLUX}

Subsection IV A reports on the experimental measurements with the diamagnetic loops. In a first setup, only Loop A was installed, and a parametric analysis on the power and xenon mass flow rate of the thruster was carried out. In a second setup, both loops A and B were used simultaneously to assess the effect of the loop diameter on the measurements.

\section{A. Variation with the power and mass flow rate}

The thruster shutdown transient lasts around $15 \mu \mathrm{s}$, the time span in which the main induced voltage of the loop is observed. Figure 9 illustrates the signal at the diamagnetic Loop A at three different powers at equal mass flow rate (left) and at three different mass flow rates at equal absorbed power (right). The plasma-induced magnetic flux $\Phi_{p}$ obtained by integrating the diamagnetic signal with Eq. (1) is shown in Fig. 10, where the data represent the averaged values between two independent samples. The repeatability of the measurements is affected by the erosion of the antenna and particle depositions at the back-plate, which are not negligible within a few hours of operation. Regarding the error bars, the difference between repeated measurements is added to the uncertainty in the signal integration procedure, which is computed as the ratio between the amplitude of the loop signal oscillations before the shutdown and the maximum amplitude registered after the shutdown. Notice that this source of uncertainty is specific of each operation point.

As it can be observed, the measured induced magnetic flux increases with microwave power. Moreover, for each tested power, a slightly higher value was found for a mass flow rate between 1 and 1.5 $\mathrm{sccm}$. Beyond this value, the induced flux decreases when increasing the mass flow rate. It should be noted, however, that the variation of $\Phi_{p}$ with the mass flow rate is small, and for the lower power values, it falls within the uncertainty of the measurements.
The behavior of $\Phi_{p}$ with power and mass flow rate is consistent with the measurements performed by Vialis et al. of the magnetic thrust in the same ECRT prototype (Fig. 6 top of Ref. 14, the measurements of direct thrust on the magnet). This correlation supports a direct relationship between the magnitude of the induced magnetic flux, as measured by the diamagnetic loop, and the magnetic thrust.

\section{B. Influence of the diamagnetic loop size}

To assess the influence of the diamagnetic loop radius, the signal at the two loops A and B was measured simultaneously. Any differences between the measurements can be attributed to each loop capturing a different plasma-induced magnetic flux $\Phi_{p}$, which in turn means the existence of $\boldsymbol{B}_{p}$ outside of the plasma beam. Contrary to the case of an infinite plasma column, where $\boldsymbol{B}_{p}$ is restricted to the inside of the plasma itself, the $2 \mathrm{D}$ plasma expansion in the $\mathrm{MN}$ is expected to generate fields that leak outside of the plasma jet. ${ }^{12}$ As such, operating two loops of different radii enables the characterization of this phenomenon.

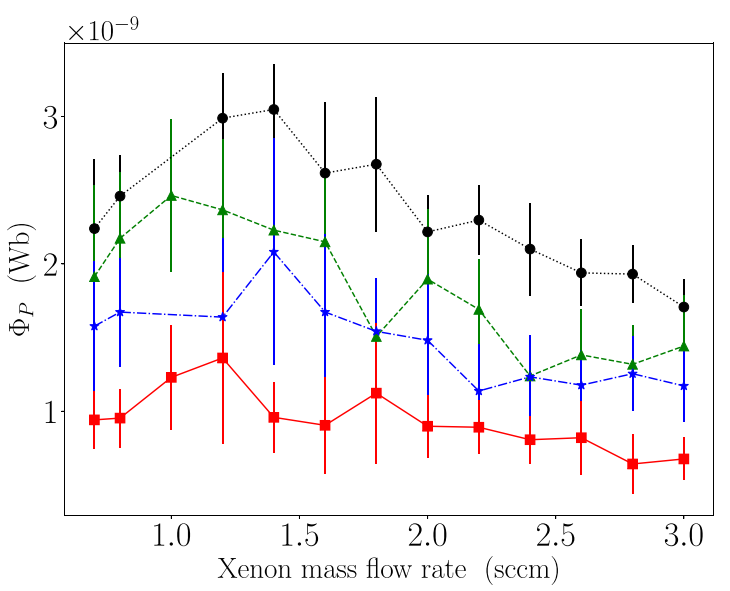

FIG. 10. Induced magnetic flux measured in Loop $A$ as a function of the mass flow rate, for different power levels: $15 \mathrm{~W}(-), 25 \mathrm{~W}(-\cdot \cdot-), 30 \mathrm{~W}(---)$, and $41 \mathrm{~W}$ (......). 
TABLE I. Experimental magnetic flux $\Phi_{p}$ from the two loops at $30 \mathrm{~W}$ of absorbed power and estimated values of mean perpendicular electron pressure inside the thruster source from the 1D and 2D models (Gaussian and polynomial initial density profiles).

\begin{tabular}{llcccc}
\hline \hline \multirow{m}{*}{$(\mathrm{sccm})$} & & $\begin{array}{c}\text { Measured } \Phi_{P} \text { at } \\
\text { shutdown }\left(10^{-9} \mathrm{~Wb}\right)\end{array}$ & $\begin{array}{c}\text { Estimated } \bar{p}_{\perp e} 1 \mathrm{D} \\
\text { model }(\mathrm{mPa})\end{array}$ & $\begin{array}{c}\text { Estimated } \\
\bar{p}_{\perp e} 2 \mathrm{D} n_{G}(0, r)(\mathrm{mPa})\end{array}$ & $\bar{p}_{\perp e} 2 \mathrm{D} n_{P}(0, r)(\mathrm{mPa})$ \\
\hline \multirow{2}{*}{$1 \mathrm{sccm}$} & Loop A & $2.46 \pm 0.5$ & $182 \pm 37$ & $218 \pm 44$ & $209 \pm 42$ \\
& Loop B & $10.0 \pm 2.2$ & $737 \pm 162$ & $99 \pm 22$ & $151 \pm 33$ \\
$2 \mathrm{sccm}$ & Loop A & $1.89 \pm 0.4$ & $140 \pm 30$ & $168 \pm 36$ & $157 \pm 36$ \\
& Loop B & $13.3 \pm 3.4$ & $979 \pm 250$ & $229 \pm 131$ & $193 \pm 48$ \\
\hline
\end{tabular}

The first column of Table I displays the measured $\Phi_{p}$ at two different mass flow rates ( 1 and $2 \mathrm{sccm} \mathrm{Xe}$ ) and $30 \mathrm{~W}$ power. In each case, the plasma-induced magnetic flux measured by Loop $\mathrm{B}$ is roughly one order of magnitude larger than that of Loop A. This difference indicates that $\boldsymbol{B}_{p}$ extends indeed outside of the plasma jet, and the $2 \mathrm{D}$ character of the expansion and the azimuthal electron currents must be taken into account to fully characterize the plasma-induced magnetic field, the operation of the device, and the generation of magnetic thrust. To explain the differences encountered by the two loops, and to relate the measurements of $\Phi_{p}$ to the perpendicular electron pressure, two models are proposed in Sec. V: a 1D plasma column model and a 2D fluid model available in the research group.

Finally, it is noted that the differences in $\Phi_{p}$ in Table I between the two mass flow rates are within the uncertainty of the measurement (also see Fig. 10), and thus, no conclusions can be drawn from these results in what regards the variation of these effects with the mass flow rate.

\section{MEAN PERPENDICULAR ELECTRON PRESSURE}

To relate the loop magnetic flux measurements to the plasma properties, it is necessary to consider a plasma model and the magnetic field it induces during thruster steady-state operation. To identify the dominant mechanisms that drive the plasma-induced magnetic flux through the loop and estimate the perpendicular electron pressure in the plasma, a simple analytical 1D model, similar to the one used in Ref. 27, is presented first. Then, a 2D model of the plasma expansion in the magnetic nozzle is used to discuss the influence of the $2 \mathrm{D}$ plasma distribution on the measurements by the two loops.

\section{A. Infinite plasma column model}

Consider an axially uniform $(\partial / \partial z=0)$, infinite, collisionless plasma column of radius $R_{p}$. The plasma is confined by a uniform, axially applied magnetic field $B_{z a}$, and the radial electric field in the plasma is assumed negligible. Electron mass is neglected with respect to ion mass, ions are assumed cold, and electrons can have different perpendicular and parallel pressures, $p_{\perp e}$ and $p_{\| e}$. Under these assumptions, the radial electron momentum balance reduces to

$$
0=-\frac{\partial p_{\perp e}}{\partial r}+j_{\theta e} B_{z a}
$$

where $j_{\theta e}=-e n u_{\theta e}$ is the azimuthal electron current density, which is the dominant contribution to the total plasma currents. ${ }^{9,12}$ Accordingly, the azimuthal component of Ampère's equation $\nabla \times \boldsymbol{B}_{p}=\mu_{0} \boldsymbol{j}$ for the axial plasma-induced magnetic field $B_{z p}$ reads

$$
-\frac{\partial B_{z p}}{\partial r}=\mu_{0} j_{\theta} \simeq \mu_{0} j_{\theta e} .
$$

Note that the radial component of the plasma-induced magnetic field, $B_{r p}$, is identically zero in this infinite plasma column configuration. Combining Eqs. (2) and (3) and integrating, note that both $p_{\perp e}$ and $B_{z p}$ are zero outside of the plasma, yields an expression for $B_{z p}$

$$
B_{z p}(r)=-\frac{\mu_{0}}{B_{z a}} \int_{r}^{R_{p}} \frac{\partial p_{\perp e}}{\partial r} d r=-\frac{\mu_{0}}{B_{z a}} p_{\perp e}(r) .
$$

The induced field is diamagnetic and opposes the applied one. The ratio of induced-to-applied magnetic field at the origin coincides with the plasma beta there

$$
\left|\frac{B_{z p}(0)}{B_{z a}(0)}\right|=\frac{\mu_{0} p_{\perp e}(0)}{B_{z a}^{2}(0)}=\beta_{0} .
$$

Integrating again Eq. (4) yields the induced magnetic flux measured by a loop with radius $R_{L}$ located around the plasma column upon plasma shutdown

$$
\Phi_{p}=-\frac{\mu_{0}}{B_{z a}} \int_{0}^{R_{L}} 2 \pi p_{\perp e} r d r=-\frac{\mu_{0}}{B_{z a}} \pi R_{P}^{2} \bar{p}_{\perp e},
$$

where $\bar{p}_{\perp e}$ is the cross-sectional mean of the perpendicular electron pressure in the plasma column. Two observations can be made. First, the induced magnetic flux scales with $\bar{p}_{\perp e}$, and it is weaker when the applied magnetic field is larger. Second, the radius $R_{L}$ of the measuring loop is irrelevant as long as $R_{L}>R_{P}$, which indeed means that any induced field in the region between $R_{P}$ and $R_{L}$ is ignored.

Equation (6) has been used to compute the mean perpendicular electron pressure $\bar{p}_{\perp e}$ at the exit section of the thruster shown in the second column of Table I. It is evident that the estimates of $\bar{p}_{\perp e}$ from the smaller Loop A and the larger Loop B are very different. This difference arises from the inadequacy of the $1 \mathrm{D}$ model to describe the present situation, where the plasma expands radially at a large angle in the MN region. Expectedly, the error of the 1D model increases with the radius of the diamagnetic loop used, as the contribution of the induced magnetic field lying between the loop and the plasma beam is more important.

As a cross-comparison with the data available in the literature, notice that the estimated perpendicular electron pressure presented in Table I is within the expected range. The direct measured thrust in this device has been reported to be hundreds of $\mu \mathrm{N}$ (both on the plasma source and on the permanent magnet). ${ }^{29}$ It is expected that the magnetic thrust is proportional to the perpendicular plasma pressure 
times an "effective" area. By taking the backplate of the thruster as a reference area, $5.73 \times 10^{-4} \mathrm{~m}^{2}$, a plasma pressure of hundreds of megapascal is obtained, which is in line with the results reported here. In parallel, the electron temperature is expected to be various tens of electronvolt $\left(20 \mathrm{eV}\right.$ have been measured in the near plume region $\left.{ }^{30}\right)$ and the mean plasma density inside the thruster has been estimated to be between 1 and $3 \times 10^{17} \mathrm{~m}^{-3}$ at 1 and 2.5 xenon sccm, respectively. These values were estimated by performing angular scans of current with a Faraday gridded probe and identifying the mean ion velocity at the exit plane with a simple $1 \mathrm{D}$ laser induced fluorescence setup. ${ }^{31}$ With these data, the mean plasma pressure should also be hundreds of megapascal.

\section{B. 2D plasma model}

The simple 1D model from Sec. V A fails to explain the differences in measured flux between the two loops of different radii, and it can only be regarded as a rough estimate of the induced field generated by the plasma of the ECRT. The $2 \mathrm{D}$ features of the plasma, such as the finite size of the thruster and the expansion in the magnetic nozzle need to be taken into account in the model to improve the computation of the radial mean of the perpendicular electron pressure, $\bar{p}_{\perp e}$.

The thruster itself is modeled as an axially uniform finite column of plasma in equilibrium with an assumed radial profile, from where the azimuthal current density can be readily obtained. The computation of the azimuthal current in the divergent $\mathrm{MN}$ is afforded by the $2 \mathrm{D}$ model of Ahedo and Merino (DIMAGNO). ${ }^{9}$ The model consists of the fluid equations for hot electrons and single-charged ions, which are integrated with the method of characteristics downstream from the thruster exit plane, where the plasma radial profile coincides with that in the thruster. This model has been used in the past to identify the plasma acceleration and thrust generation mechanisms in the magnetic nozzle and to explain the plasma-induced magnetic field and the plasma detachment downstream. ${ }^{11,12,32}$ In addition to the assumptions of the simple model of Sec. V A, electrons are treated as a fully magnetized, isothermal species $\left(T_{e}=T_{e 0}=\right.$ const), which, while ignoring electron anisotropy, is a reasonable approximation in the near-region of the magnetic nozzle. ${ }^{33}$ Ions, on the other hand, are only partially magnetized.

Once $j_{\theta e}$ has been computed in the whole plasma domain, it is interpolated into a regular Cartesian grid and the analytical solution of a thin current loop at each node $n$ of the grid is used to calculate the resulting plasma-induced magnetic field $\boldsymbol{B}_{p}$

$$
\begin{aligned}
B_{z p}= & \sum_{n} \frac{\mu_{0} I_{n}}{2 \pi} \frac{1}{\sqrt{\left(r+r_{n}\right)^{2}+\left(z-z_{n}\right)^{2}}} \\
& \times\left[\mathrm{K}\left(m_{n}\right)-\frac{r^{2}-r_{n}^{2}+\left(z-z_{n}\right)^{2}}{\left(r-r_{n}\right)^{2}+\left(z-z_{n}\right)^{2}} \mathrm{E}\left(m_{n}\right)\right], \\
B_{r p}= & -\sum_{n} \frac{\mu_{0} I_{n}}{2 \pi r} \frac{\left(z-z_{n}\right)}{\sqrt{\left(r+r_{n}\right)^{2}+\left(z-z_{n}\right)^{2}}} \\
& \times\left[\mathrm{K}\left(m_{n}\right)-\frac{r^{2}+r_{n}^{2}+\left(z-z_{n}\right)^{2}}{\left(r-r_{n}\right)^{2}+\left(z-z_{n}\right)^{2}} \mathrm{E}\left(m_{n}\right)\right],
\end{aligned}
$$

where $\mathrm{K}(m)$ and $\mathrm{E}(m)$ are the complete elliptic integrals of the first and second kinds, ${ }^{34} z_{n}, r_{n}$, and $I_{n}$ are the axial position, radial position, and associated azimuthal current of node $n$, and

$$
m_{n}=\frac{4 r_{n} r}{\left(r+r_{n}\right)^{2}+\left(z-z_{n}\right)^{2}} .
$$

The resulting 2D model of the plasma expansion depends on two dimensionless parameters. First, there is the normalized ion gyrofrequency computed with the applied magnetic field at the origin $B_{a 0}$, $\hat{\Omega}_{i 0}=e B_{a 0} R_{p} / \sqrt{m_{i} T_{e 0}}$, which controls the ion magnetization strength and only plays a minor role in the near-field expansion. ${ }^{12}$ In the present thruster, with $B_{a 0}=0.0558 \mathrm{~T}$, xenon as propellant, and an electron temperature in the range $20-30 \mathrm{eV}$, one has $\hat{\Omega}_{i 0} \simeq 0.13{ }^{30}$ Second, there is the plasma beta parameter at the origin, $\beta_{0}$, which is calculated so that the magnetic flux through the diamagnetic loop area matches the experimental measured value. Finally, the model depends on the initial plasma profile at the thruster, which is currently not known. To evaluate the influence of the initial plasma profile on the computation of $\bar{p}_{\perp e}$ from the measurement of $\Phi_{p}$ at the loop, two different profiles (a Gaussian one and a polynomial one) have been simulated to assess the influence of this aspect on the measured magnetic flux. Figure 11 shows the plasma pressure $p$ and azimuthal electron current density $j_{\theta e}$ of the two considered initial profiles, normalized with their mean perpendicular electron pressure $\bar{p}_{\perp e}$ ). The azimuthal current is larger where pressure gradients are larger and thus varies between the two profiles. The Gaussian profile has larger currents near the axis, whereas in the more radially uniform polynomial case, $j_{\theta e}$ is negligible except near the plasma edge.

Figure 12 shows the $2 \mathrm{D}$ maps of the resulting plasma-induced magnetic field $B_{p}$ and the developed azimuthal electron currents along the expansion $j_{\theta e}$ that result from the Gaussian and polynomial initial profiles. As the plasma currents downstream from the simulation box have an impact in the domain, the DIMAGNO simulations have been extended down to $z=60 \mathrm{~cm}$ but results are retained only down to $z=25 \mathrm{~cm}$.

The computed plasma-induced magnetic field from Eqs. (7) and (8) is integrated and averaged along the measuring loop section and equated to the measured magnetic flux. The resulting expressions allow computing $\bar{p}_{\perp e}$ at the thruster exit plane, which is shown in the last two columns of Table I. Comparing these values with those of the 1D model of Sec. V A, several remarks can be made. First, the difference in the estimated value of $\bar{p}_{\perp e}$ computed using the two models (irrespective of the initial profile used) is small for the smaller Loop A. This suggests that $2 \mathrm{D}$ effects are not essential near the thruster axis, but become important away from it, and that the $1 \mathrm{D}$ model can be used to estimate $\bar{p}_{\perp e}$ when the loop radius is close to the plasma radius. Second, it is noted that the $2 \mathrm{D}$ model provides a far better agreement with the $\bar{p}_{\perp e}$ estimated with the two loops than the $1 \mathrm{D}$ model: while there is a discrepancy larger than the uncertainty in the measurements, it is evident that the 2D model produces values in the same range with each loop, whereas the 1D model fails by one order of magnitude. This indicates that $2 \mathrm{D}$ effects are important in the estimation of $\bar{p}_{\perp e}$ when the loop radius is large. Concerning the sensitivity to the initial plasma density profile in the $2 \mathrm{D}$ model, data show that it only causes a difference for the larger Loop B, while its effect is negligible for Loop A. While it is not possible to assert with certainty which profile fits better with the real plasma beam, the polynomial profile seems to provide a slightly better agreement between the estimate of $\bar{p}_{\perp e}$ from Loop A and B data than the Gaussian profile. Additional investigation of the radial plasma profile (possibly with other plasma diagnostics) is necessary to confirm this observation. 
(a)

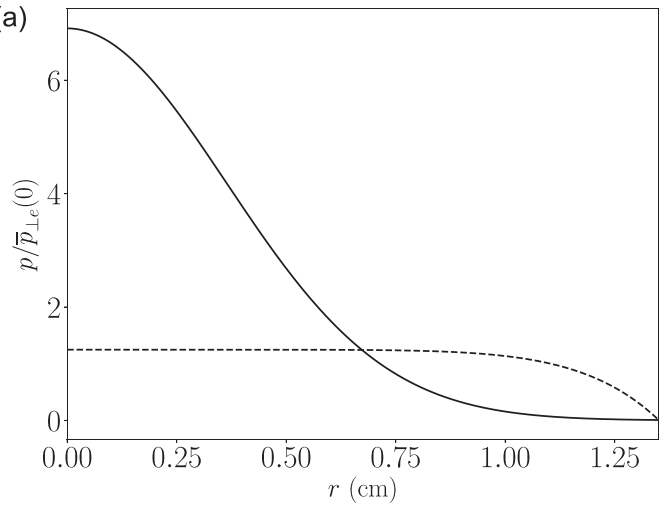

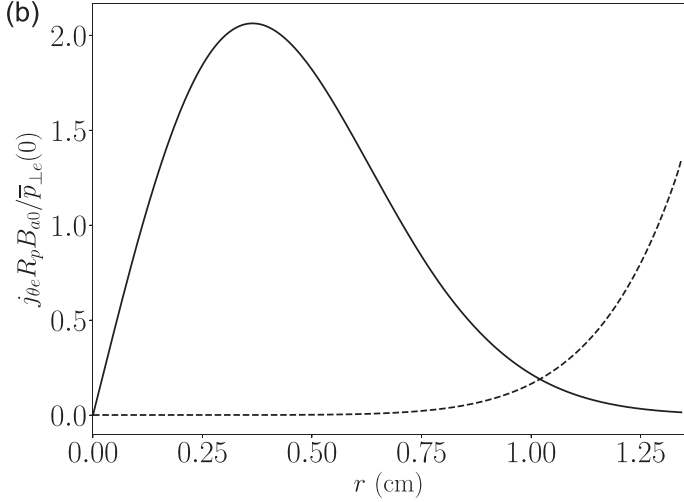

FIG. 11. (a) Normalized plasma pressure $p / \bar{p}_{\perp e}(0)$ and $(b)$ normalized azimuthal current density $j_{\theta e} R_{p} B_{a 0} / \bar{p}_{\perp e}(0)$ of the two considered initial plasma profiles in the thruster for the Gaussian distribution $n_{G}(0, r)=\exp \left(-3 \cdot \ln (10) \cdot r^{2}\right)(-)$ and the polynomial distribution $n_{P}(0, r)=1-r^{8}(---)$.

Finally, the $2 \mathrm{D}$ plasma model used here allows to compute the total magnetic thrust by integrating the $j_{e \theta} B_{r}$ component along the simulation domain. For instance, for the first row of Table I ( $1 \mathrm{sccm})$, the computed thrust with Loop $\mathrm{A}$ is $251 \mu \mathrm{N}$ and $207 \mu \mathrm{N}$ for the

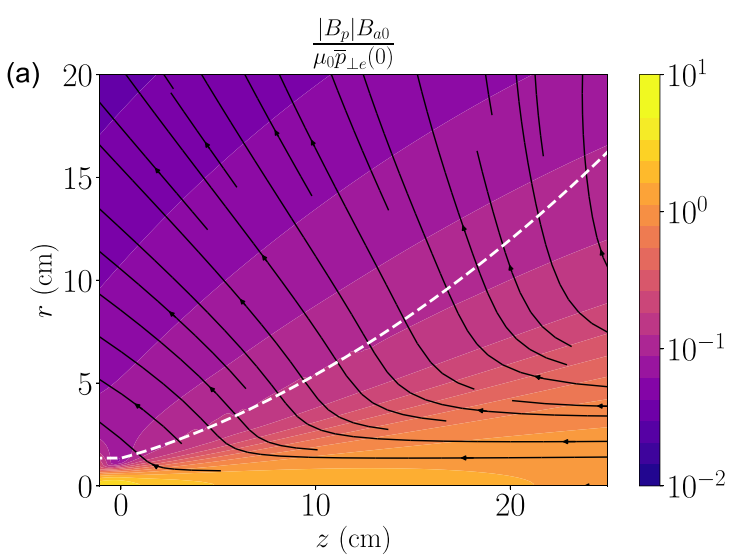

(c)

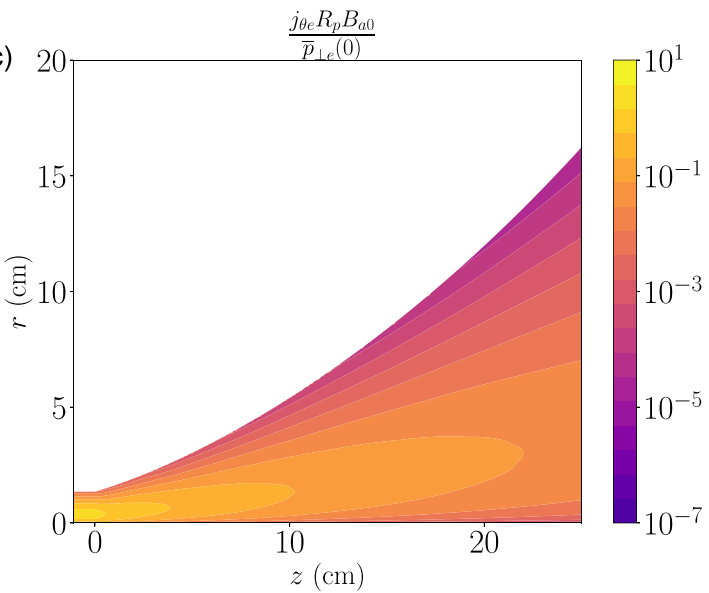

Gaussian and polynomial profiles, respectively, while for Loop B is $128 \mu \mathrm{N}$ and $170 \mu \mathrm{N}$. As a reference, the maximum azimuthal current density for the Gaussian profile at $1 \mathrm{sccm}$ is around $4000 \mathrm{~A} / \mathrm{m}^{2}$. The radial magnetic field at this point is 31.4 Gauss. With these values, a
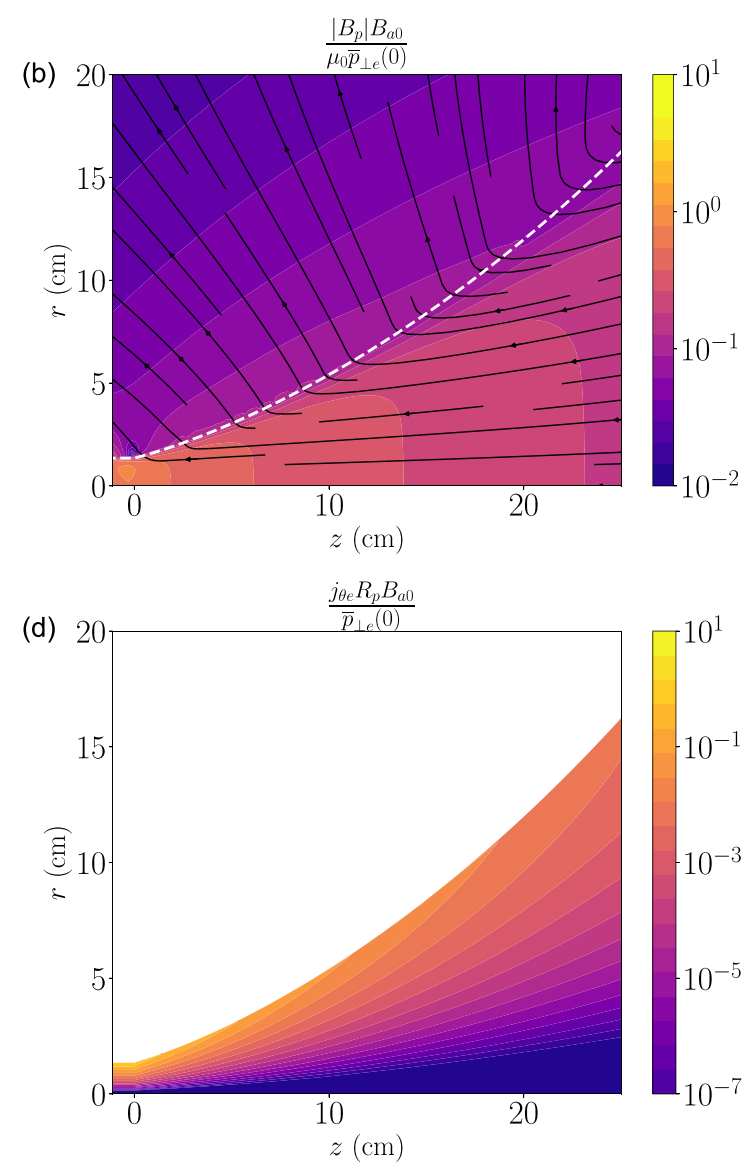

FIG. 12. Plasma-induced magnetic field and azimuthal electron current density in the near-region plume for the Gaussian (a) and (c) and polynomial (b) and (d) initial plasma profiles. The white dashed line represents the plasma boundary. 
magnetic thrust of a few hundreds of $\mu \mathrm{N}$ is estimated. These values of thrust are on the order of the values given in the literature ${ }^{29}$ even though the experimental setup given in the study of Ref. 29 was different from the one presented here, including different vacuum facilities and losses through the power line.

\section{CONCLUSION}

Two diamagnetic loops of different radii have been employed to measure non-intrusively the plasma-induced magnetic flux $\Phi_{p}$ at the exit plane of an Electron Cyclotron Plasma Thruster prototype at different power levels and propellant mass flow rates. The proposed setup has demonstrated to be capable of detecting the plasma-induced magnetic flux by integrating the diamagnetic signal in the loop during thruster shutdown.

The calibration of the diamagnetic loops assessed possible sources of error when applying this technique to an ECRT. In particular, it identified the influence of eddy currents in the thruster walls as a major source of error depending on their material, and it was found that graphite results in a cleaner diamagnetic signal than aluminum, maintaining the thruster operation conditions unchanged. Plasma oscillations of the loop signal during steady state operation were seen to be large, and they induce uncertainty in the measurements. Characterizing and understanding the source of these oscillations is interesting by itself and will be the subject of future work.

The diamagnetic flux $\Phi_{p}$ generated by the plasma increases with applied power and has a weak non-monotonic behavior with the mass flow rate. These trends agree well with those of the recent direct measurements of magnetic thrust by Vialis et al. in the same thruster (in a different facility and with different diagnostics). Both the diamagnetic flux $\Phi_{p}$ and the magnetic thrust are directly related to the perpendicular electron pressure in the plasma, which in a thruster with anisotropic heating like the ECRT may differ from the parallel electron pressure.

The magnetic flux measured by the larger loop has been found to be significantly greater than the magnetic flux measured by the smaller one. This attests to the importance of the $2 \mathrm{D}$ geometry of the plasmainduced magnetic field, as the magnetic flux created by the plasma spills outside of the plume volume, meaning that a larger loop will capture more flux. Two plasma models, $1 \mathrm{D}$ and $2 \mathrm{D}$, have been used to simulate $\Phi_{p}$ and relate its value to the radial mean of the perpendicular electron pressure at the thruster exit plane, $\bar{p}_{\perp e}$, showing that it is within the expected range. Electron pressures up to $200 \mathrm{mPa}$ have been inferred from the diamagnetic loop measurements, which are in line with previous measurements of electron temperature, plasma density, and thrust reported in the literature for this thruster. ${ }^{29-31}$ The $1 \mathrm{D}$ model only provides valid estimates of $\bar{p}_{\perp e}$ for the loop of smaller radii, and the necessity of a $2 \mathrm{D}$ model of the plasma becomes apparent when the larger diamagnetic loop is used. These results point out the limitations of the simple formulas emanating from the $1 \mathrm{D}$ model to compute the electron pressure in the plasma.

In order to assess the effect of the radial plasma profile in the thruster in the 2D model, two different ones were tested. The differences observed between the two are small, especially when using the smaller loop, but it does have an impact with the larger one. Therefore, the smaller loop is a better option for an estimation of the plasma pressure at the source.
Finally, neither model accounts explicitly for the anisotropy of the electron population; future research must advance in this direction, and in general, improve the electron model used in order to refine the estimates of this work. This may be especially relevant for the ECRT for the reasons stated above.

\section{ACKNOWLEDGMENTS}

This work was made in the framework of project MINOTOR that has received funding from the European Union's Horizon 2020 research and innovation program under Grant agreement No 730028. Additional funding came from the Spanish R\&D National Plan (Grant No. PN ESP2016-75887).

\section{REFERENCES}

${ }^{1}$ E. F. Gibbons and D. B. Miller, "Experiments with an electron cyclotron resonance plasma accelerator," AIAA J. 2(1), 35-41 (1964).

${ }^{2}$ M. Nagatomo, "Plasma acceleration by high frequency electromagnetic wave in staticmagnetic field gradient," in 6th Electric Propulsion and Plasmadynamics Conference (American Institute of Aeronautics and Astronautics, September 1967), p. 660.

${ }^{3}$ J. Sercel, "Electron-cyclotron-resonance (ECR) plasma thruster research," in 24th Joint Propulsion Conference (American Institute of Aeronautics and Astronautics, July 1988), p. 2916.

${ }^{4}$ E. Bickford Hooper, B. W. Stallard, and M. A. Makowski, "Whistler wave driven plasma thruster,” AIP Conf. Proc. 271, 1419-1424 (1993).

${ }^{5}$ J. Jarrige, P.-Q. Elias, D. Packan, and F. Cannat, "Characterization of a coaxial ECR plasma thruster," in 44th AIAA Plasmadynamics and Lasers Conference (American Institute of Aeronautics and Astronautics, June 2013), p. 2628.

${ }^{6} \mathrm{C}$. Charles and R. Boswell, "Current-free double-layer formation in a highdensity helicon discharge,” Appl. Phys. Lett. 82(9), 1356-1358 (2003).

${ }^{7}$ D. Pavarin, F. Ferri, M. Manente, D. Curreli, Y. Guclu, D. Melazzi, D. Rondini, S. Suman, J. Carlsson, C. Bramanti et al., "Design of $50 \mathrm{~W}$ helicon plasma thruster," in 31st International Electric Propulsion Conference, Ann Arbor, MI, 2009, pp. 2009-2205.

${ }^{8}$ J. Navarro-Cavallé, M. Wijnen, P. Fajardo, and E. Ahedo, "Experimental characterization of a $1 \mathrm{~kW}$ Helicon plasma thruster," Vacuum 149, 69-73 (2018).

${ }^{9} \mathrm{E}$. Ahedo and M. Merino, "Two-dimensional supersonic plasma acceleration in a magnetic nozzle," Phys. Plasmas 17(7), 073501 (2010).

${ }^{10} \mathrm{M}$. Merino and E. Ahedo, "Magnetic nozzles for space plasma thrusters," Encyclopedia of Plasma Technology, edited by J. L. Shohet (Taylor and Francis, 2016), Vol. 2, pp. 1329-1351.

${ }^{11}$ M. Merino and E. Ahedo, "Plasma detachment in a propulsive magnetic nozzle via ion demagnetization,” Plasma Sources Sci. Technol. 23(3), 032001 (2014).

${ }^{12} \mathrm{M}$. Merino and E. Ahedo, "Effect of the plasma-induced magnetic field on a magnetic nozzle,” Plasma Sources Sci. Technol. 25(4), 045012 (2016).

${ }^{13}$ R. Geller, Electron Cyclotron Resonance Ion Sources and ECR Plasmas (CRC Press, 1996).

${ }^{14}$ T. Vialis, J. Jarrige, and D. Packan, "Separate measurements of magnetic and pressure thrust contributions in a magnetic nozzle electron cyclotron resonance plasma thruster," in Space Propulsion Conference, No. 499, Seville, Spain, May 2018.

${ }^{15}$ I. H. Hutchinson, "Principles of plasma diagnostics: Second edition," Plasma Phys. Controlled Fusion 44(12), 2603-2603 (2002).

${ }^{16}$ W. M. Hooke, M. A. Rothman, J. Sinnis, and J. Adam, "Temperature and power measurements in a plasma heated by absorption of ion cyclotron eaves," Phys. Fluids 8(6), 1146 (1965).

${ }^{17} \mathrm{~K}$. Uo, "Perpendicular temperature of the C-Stellarator plasma," Phys. Fluids 8(2), 384 (1965).

${ }^{18}$ S. Yoshikawa, M. A. Rothman, and R. M. Sinclair, "Absorption of ion cyclotron waves by one component of a two-ion plasma," Phys. Rev. Lett. 14(7), 214-216 (1965).

${ }^{19}$ J. C. Sercel, "An experimental and theoretical study of the ECR plasma engine," Ph.D. thesis (California Institute of Technology, 1993). 
${ }^{20}$ A. Ando, M. Inutake, K. Hattori, M. Shibata, and Y. Kasashima, "ICRF heating and plasma acceleration with an open magnetic field for the advanced space thruster," Fusion Sci. Technol. 51(2T), 72-74 (2007).

${ }^{21}$ C. Thomas, N. Gascon, and M. Cappelli, "Nonintrusive characterization of the azimuthal drift current in a coaxial E cross B discharge plasma," Phys. Rev. E 74(5), 056402 (2006)

${ }^{22}$ M. Prioul, A. Bouchoule, S. Roche, L. Magne, D. Pagnon, M. Touzeau, and P. Lasgorceix, "Insights on physics of Hall thrusters through fast current interruptions and discharge transients," in 27th International Electric Propulsion Conference, IEPC-01-059, 2001.

${ }^{23} \mathrm{~K}$. Takahashi, A. Chiba, A. Komuro, and A. Ando, "Experimental identification of an azimuthal current in a magnetic nozzle of a radiofrequency plasma thruster," Plasma Sources Sci. Technol. 25(5), 055011 (2016).

${ }^{24}$ B. R. Roberson, R. Winglee, and J. Prager, "Enhanced diamagnetic perturbations and electric currents observed downstream of the high power helicon," Phys. Plasmas 18(5), 053505 (2011).

${ }^{25}$ R. L. Stenzel and J. M. Urrutia, "Electron magnetohydrodynamic turbulence in a high-beta plasma. I. Plasma parameters and instability conditions," Phys. Plasmas 7(11), 4450-4456 (2000).

${ }^{26}$ C. S. Corr and R. W. Boswell, "High-beta plasma effects in a low-pressure helicon plasma,” Phys. Plasmas 14(12), 122503 (2007).

${ }^{27}$ J. Noland, O. Tarvainen, J. Benitez, D. Leitner, C. Lyneis, and J. Verboncoeur, "Studies of electron heating on a $6.4 \mathrm{GHz}$ ECR ion source through measurement of diamagnetic current and plasma bremsstrahlung," Plasma Sources Sci. Technol. 20(3), 035022 (2011).

${ }^{28}$ J. Booske, M. McCarrick, S. Douglass, J. Paquette, R. Ellis, and W. Getty, "Frequency compensation of a diamagnetic loop using a digital data acquisition system,” J. Phys. E: Sci. Instrum. 20(6), 627 (1987).

${ }^{29} \mathrm{~T}$. Vialis, J. Jarrige, A. Aanesland, and D. Packan, "Direct thrust measurement of an electron cyclotron resonance plasma thruster," J. Propul. Power 34(5), 1323-1333 (2018).

${ }^{30}$ T. Lafleur, F. Cannat, J. Jarrige, P. Elias, and D. Packan, "Electron dynamics and ion acceleration in expanding-plasma thrusters," Plasma Sources Sci. Technol. 24(6), 065013 (2015).

${ }^{31}$ S. Correyero Plaza, J. Jarrige, D. Packan, and E. Ahedo Galilea, "Measurement of anisotropic plasma properties along the magnetic nozzle expansion of an electron cyclotron resonance thruster," in 35th International Electric Propulsion Conference, No. IEPC-2017-437, Electric Rocket Propulsion Society, Fairview Park, OH, Atlanta, GA, 2017.

${ }^{32} \mathrm{M}$. Merino and E. Ahedo, "Fully magnetized plasma flow in a magnetic nozzle," Phys. Plasmas 23(2), 023506 (2016).

${ }^{33} \mathrm{M}$. Merino and E. Ahedo, "Influence of electron and ion thermodynamics on the magnetic nozzle plasma expansion,” IEEE Trans. Plasma Sci. 43, 244-251 (2015).

${ }^{34}$ M. Abramowitz and I. A. Stegun, Handbook of Mathematical Functions: With Formulas, Graphs, and Mathematical Tables (Courier Corporation, 1965), Vol. 55. 